\title{
Treatment with a herbal formula B40I enhances neuroprotection and angiogenesis in the R6/2 mouse model of Huntington's disease
}

This article was published in the following Dove Press journal:

Drug Design, Development and Therapy

16 February 2015

Number of times this article has been viewed

\author{
Sheue-Er Wang' \\ Ching-Lung Lin' \\ Chih-Hsiang Hsu' \\ Shuenn-Jyi Sheu ${ }^{2}$ \\ Chiang-Ting Chien' \\ Chung-Hsin $\mathrm{Wu}^{\prime}$ \\ 'Department of Life Science, \\ National Taiwan Normal University, \\ ${ }^{2}$ Brion Research Institute of Taiwan, \\ Taipei, Taiwan
}

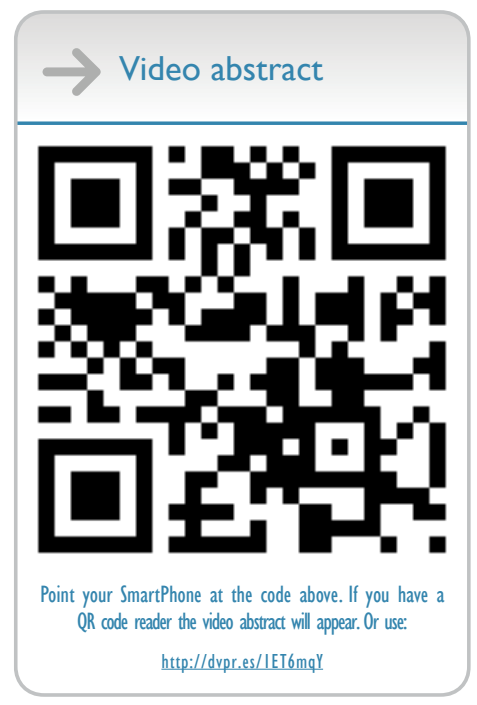

Correspondence: Chung-Hsin Wu Department of Life Science, National Taiwan Normal University, 88, Ting-Chou Road, Section 4, Taipei II677, Taiwan Tel +8862 77346363

Fax +886 2293I 2904

Email megawu@ntnu.edu.tw

\begin{abstract}
Huntington's disease (HD) is a neurodegenerative disease characterized by motor dysfunction and early death. Despite years of research, the mechanisms responsible for chronic neurodegeneration of HD remain elusive. Chinese traditional medicines might provide new insights or new therapy for HD. The Chinese herbal formula B401 is a well-known Taiwan-US patent formula and a health supplement for promoting blood circulation and enhancing brain function. This study aimed to elucidate the neuroprotective effects of the Chinese herbal formula B401 on the syndrome of HD. Then, we compared the life span and body weight of R6/2 HD mice with and without oral B401 treatment. The ameliorative effects of B401 on the symptom of HD mice were investigated through behavior tests. Expressions of proteins for neuroprotection, angiogenesis, and inflammation in the brain tissue of R6/2 HD mice were compared by using immunostaining and Western blotting techniques. Our study in vitro showed that viabilities of glutamate-treated SH-SY5Y cells were significantly increased under B401 treatment. Our results in vivo showed that duration of survival was increased, body weight loss was reduced, and motor ability was improved in R6/2 HD mice under oral B401 treatment. Subcutaneous microcirculation was enhanced in 3-month R6/2 HD mice under intraperitoneal B401 injections as observed by using moorFLPI laser Doppler imager. Atrophy of cerebrum, midbrain, and cerebellum in 3-month R6/2 HD mice under oral B401 treatment was alleviated as observed by utilizing magnetic resonance imaging. Evidence from immunostaining and Western blotting analysis showed that expressions of mutant huntingtin and tumor necrosis factor-alpha were reduced, while expressions of brain-derived neurotrophic factor and vascular endothelial growth factor were enhanced in the brain tissue of 2-month R6/2 HD mice under oral B401 treatment. We suggest that the herbal formula B401 can be developed as a medical supplement for ameliorating neurodegenerative diseases of HD via reducing mutant huntingtin aggregation and excitotoxicity, enhancing neuroprotection and angiogenesis, and alleviating inflammation in the brain.
\end{abstract}

Keywords: Huntington's disease, traditional Chinese medicine, neuroprotection, excitotoxicity, angiogenesis, inflammation, R6/2 mouse model

\section{Highlights}

1. We report anti-huntingtin aggregation potential of the Chinese herbal formula B401 in the brain of R6/2 mouse model of Huntington's disease (HD).

2. The Chinese herbal formula B401 upregulates brain-derived neurotrophic factor and vascular endothelial growth factor, and suppresses tumor necrosis factor-alpha in the brain of R6/2 HD mice.

3. The Chinese herbal formula B401 may serve as a potential therapeutic strategy for HD brain atrophy. 


\section{Introduction}

The cause of Huntington's disease (HD) relates to an expanded CAG repeat in exon 1 of the huntingtin gene. ${ }^{1,2}$ Under normal circumstances, huntingtin is the key to brain anti-apoptosis. ${ }^{3-5}$ It also prevents the production of mutant protein. ${ }^{6,7}$ Once the genetic disorder takes place, it leads to cortical and striatal degeneration, and gathers mutant huntingtin. ${ }^{1}$ The outcome is the mutant and dysfunctional protein. ${ }^{8}$ Although the mutant protein can be found in the patients' pathogenetic basal ganglia neurons, its connection with the pathology of HD remains obscure., ${ }^{9} 10$ The dilemma of conventional pharmacological treatment has prompted the search for alternative treatments for HD. One such treatment is the use of herbal medicines. Traditional herbal medicine may offer therapies with better efficacy and fewer side effects. Therefore, herbal treatment is being preferred over conventional treatments. Much attention and scope are drawn toward herbal remedy of many brain disorders. Studies have shown the therapeutic potential of chronic treatment with several herbal medicines in various neurodegenerative diseases, such as Alzheimer's disease (AD), Parkinson's disease (PD), and HD. ${ }^{11-13}$ Chronic treatment with these herbal medicines was shown to have neuroprotective effect to normalize brain transmitter levels and to increase brainderived neurotrophic factor (BDNF) levels.

Transgenic mouse models of HD have been used for promoting the study of HD. ${ }^{14}$ One of the most popular models is the R6/2 HD mouse. It carries $150 \mathrm{CAG}$ repeats and expresses exon 1 of the human HD gene. ${ }^{15}$ In this study, R6/2 HD mouse is chosen as the model because of its practical use for testing HD therapies. ${ }^{16}$ It shows a progressive homogeneous HD-like phenotype. During its life cycle from 14 weeks to 16 weeks, the R6/2 HD mouse gradually suffers from neurological movement disorder, declined motor performance, and reducing body weight until death. ${ }^{14}$

In Taiwan, the Chinese herbal formula B401 has been developed as a health supplement for promoting blood circulation and enhancing brain function. Thus, we assumed that B401 might have neuroprotective effects on HD. Here, we examined the neuroprotective effects of the B401, assessing neuroblastoma SH-SY5Y cell viability in vitro, and using the HD-like R6/2 HD mouse model to examine motor behavior, microvascular blood flow, brain morphology, inflammation, and mutant huntingtin aggregation under B401 treatment.

\section{Materials and methods}

\section{Chromatographic fingerprint analysis for the Chinese herbal formula B40I}

Originally developed as a health supplement, the herbal formula B401 (supplied by Sun-Ten Pharmaceutical Company) contains Panax ginseng, Astragalus membranaceus, Angelica sinensis, Rehmannia glutinosa, Ligustri fructus, and Eclipta prostrata. Figure 1A shows the liquid chromatographic charts representing the wavelengths of these six ingredients. The marker compounds of these ingredients are ginsenosides Rb1, formononetin, 5-hydroxymethylfurfural, ferulic acid, oleanolic acid, and wedelolactone individually. All chemicals used in production were analytical and solubilized in distilled $\mathrm{H}_{2} \mathrm{O}$ / $\mathrm{MeOH}$. The production also depended on high-performance liquid chromatography grade acetonitrile from Burdick \& Jackson (Gyeonggi-do, Korea) and methanol from Avantor (Center Valley, PA, USA). Moreover, purified water was provided by Milli-Q water purification system (EMD Millipore, Billerica, MA, USA). An additional information is that the experiments would not be affected by vehicle.

\section{SH-SY5Y cell viability assay}

As shown by a previous study, ${ }^{17}$ we cultured human neuroblastoma SH-SY5Y cells in culture flasks $\left(25 \mathrm{~cm}^{2}\right)$. Then, SH-SY5Y cells were maintained in Dulbecco's Modified Eagle's Medium (DMEM) and incubated at $37^{\circ} \mathrm{C}$ in a humidified atmosphere ( $95 \%$ air and $5 \% \mathrm{CO}_{2}$ ). SH-SY5Y cells must be placed on glass-based culture dishes $(35 \mathrm{~mm})$ no later than 24 hours before the experiments. The cultured neuroblastoma SH-SY5Y cell lines go through $500 \mathrm{nM}$ retinoic acid (RA) treatments for 18 hours and undergo neuronal differentiation over 3-10 days. Experiments were conducted in laboratory environment. The cells were cultured with and without Chinese herbal formula B401 (10-30 mg/mL dissolved in dimethyl sulfoxide) or $N$-methyl-D-aspartate (NMDA) receptor antagonist, MK-801 $(10 \mu \mathrm{M})$ for 1 hour, and then treated with glutamate $(10 \mathrm{mM})$ for 24 hours. Control cultures were also administered the same amount of DMEM. After the treatment with B401 and glutamate, we added $0.5 \mathrm{mg} / \mathrm{mL}$ 3-(4,5-dimethylthiazol-2yl)-2,5-diphenyltetrazolium bromide (MTT) to culture media. SH-SY5Y cells were incubated for 3 hours at $37^{\circ} \mathrm{C}$. Then, absorbance was read at an optical density (OD) of $570 \mathrm{~nm}$ with an enzyme-linked immunosorbent assay reader (uQuant, Bio-Tek Inc, Winooski, Vermont, USA). The percentage of cell viability was calculated as OD at $570 \mathrm{~nm}$ of experiment well divided by OD at $570 \mathrm{~nm}$ of control well. All treatments were repeated at least three times under each condition.

\section{R6/2 transgenic HD mice preparation and monitoring}

Among all the HD animal models, the transgenic R6/2 mouse (B6CBA-Tg(HDexon1)62Gpb/1 J) is widely acclaimed because of its usefulness. ${ }^{13}$ Life cycle of R6/2 HD mice is estimated to be $12-16$ weeks. Their neurological phenotype was progressed with age, including choreiform-like or 
A

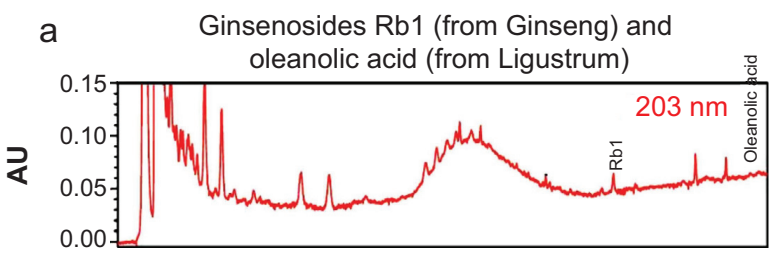

d

Ferulic acid (from Ligustrum)

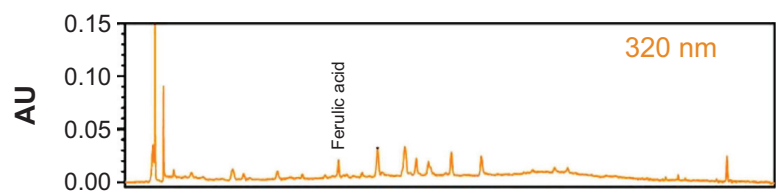

b

Formononetin (from Astragalus)

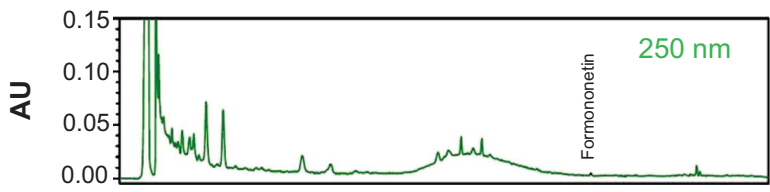

e

Wedelolactone (from Eclipta)
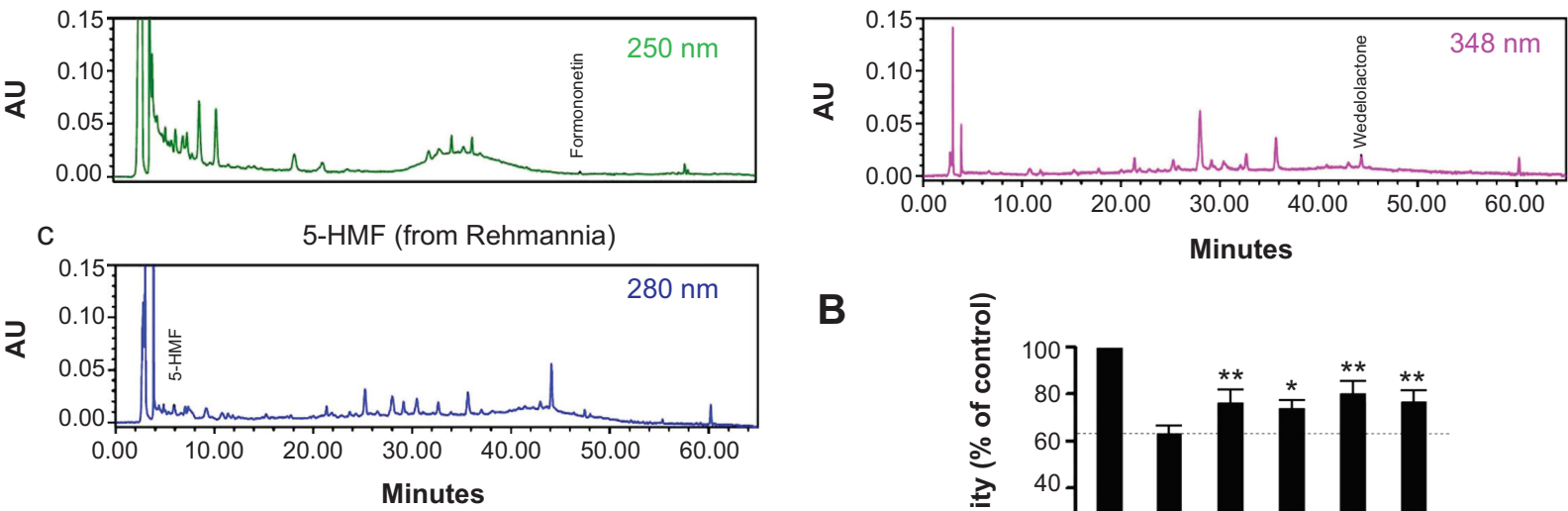

B

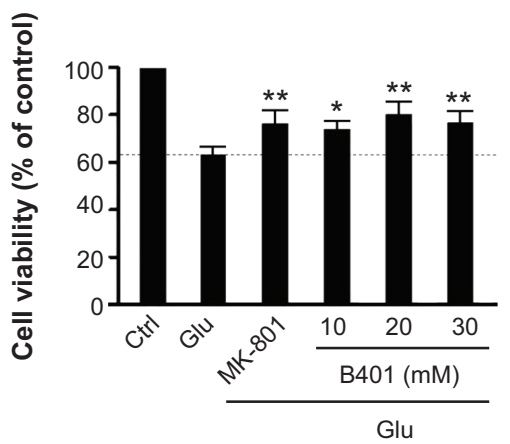

Figure I Chromatographic fingerprint analysis and cell viability assay for the Chinese herbal formula B40I.

Notes: (A) HPLC fingerprint of the herbal formula B40I. Characteristic peaks of B40I, ie, (a) ginsenosides RbI (molecular formula: $\mathrm{C}_{54} \mathrm{H}_{92} \mathrm{O}_{23} ;$ molecular weight: I, $109.3 \mathrm{~g} / \mathrm{mol}$; from Panax ginseng) and oleanolic acid (molecular formula: $\mathrm{C}_{30} \mathrm{H}_{48} \mathrm{O}_{3}$; molecular weight: $456.7 \mathrm{~g} / \mathrm{mol}$; from Ligustri fructus), (b) formononetin (molecular formula: $\mathrm{C}_{16} \mathrm{H}_{12} \mathrm{O}_{4}$; molecular weight: $268.3 \mathrm{~g} / \mathrm{mol}$; from Astragalus membranaceus), (c) 5-hydroxymethylfurfural (molecular formula: $\mathrm{C}_{6} \mathrm{H}_{6} \mathrm{O}_{3}$; molecular weight: $126.1 \mathrm{~g} / \mathrm{mol}$; from Rehmannia glutinosa), (d) ferulic acid (molecular formula: $\mathrm{C}_{10} \mathrm{H}_{10} \mathrm{O}_{4}$; molecular weight: $194.2 \mathrm{~g} / \mathrm{mol}$; from L. fructus), (e) wedelolactone (molecular formula: $\mathrm{C}_{16} \mathrm{H}_{10} \mathrm{O}_{7}$; molecular weight: $314.3 \mathrm{~g} / \mathrm{mol}$; from Eclipta prostrata), were identified and marked at the corresponding peaks in the fingerprint. (B) Cell viabilities of RA-induced SH-SY5Y cells with glutamate treatment for 24 hours in the absence or presence of the B40I at indicated doses, or NMDA receptor antagonist, MK-80I. Glutamate-treated SH-SY5Y cell viabilities were significantly increased under B40I (B40I, $n=6$ for each B40I dose treatment) and MK-80I treatment (MK-80I, $n=6$ ) individually than their control $(n=6)$. Values are mean \pm SEM ( $* * P<0.01, * P<0.05$, two-way ANOVA followed by a Student-Newman-Keuls multiple comparisons posttest).

Abbreviations: AU, arbitrary perfusion units; 5-HMF, 5-hydroxymethylfurfural; Ctrl, control; Glu, glutamate; HPLC, high-performance liquid chromatography; RA, retinoic acid; NMDA, N-methyl-D-aspartate; SEM, standard error of the mean; ANOVA, analysis of variance.

unintentional movements, tremor, and epilepsy. They may also suffer from muscle wasting and premature death. Some of the phenotypes are similar to the quality of HD, like movement disorders, uncommon vocalization, and loss of muscle. In addition, huntingin protein could be found in neuronal intranuclear inclusions. During the 12-hour light/dark cycle, their supplement of food and water is unrestrained. The body weights of experimental mice were monitored daily. All the mice in the experiment were checked daily for determining life span. Their death judgment rests on cardiac arrest. Mice euthanasia would be carried out under the circumstance of moribund, including lack of movement even after prodding, and/or lying on the side and lack of righting reflex. All mice in the experiment were maintained according to the international guidelines for care and use of laboratory animals. Experimental procedures involving animals were approved by the Committee on Animal Research of National
Taiwan Normal University (NTNU) and implemented under the guidelines of the Committee (protocol number: NTNU/ Animal Use/No 13017/Nov 26, 2013).

\section{Oral B40I treatments in $\mathrm{R} 6 / 2$ mice}

In this study, we chose 2-month R6/2 HD mice for oral treatment with either B401 extract $(30 \mathrm{mg} / \mathrm{mL}$, the $\mathrm{pH}$ value was close to 7.0) or its vehicle (dimethyl sulfoxide) in the drinking water. Twice weekly, animals were weighed, and their water was refreshed. All doses were adjusted according to individual weight and water consumption, which averaged $30 \mathrm{~mL} /$ day. On the other hand, we chose 3-month R6/2 HD mice for intraperitoneal injection of B401 extract $(100 \mathrm{mg} / \mathrm{kg}$, the $\mathrm{pH}$ value was close to 7.0), or its vehicle (dimethyl sulfoxide) in saline solution. The dosage and administration of B401 extract in R6/2 HD mice were much lower than the dosage of $\mathrm{IC}_{50}$. All doses were adjusted according to individual weight, 
which averaged $10 \mathrm{~mL} / \mathrm{kg}$. According to our observations, R6/2 HD mice began to develop HD symptoms at the age of 8 weeks and began to die at the age of 14 weeks. Thus, we chose 2-month R6/2 HD mice to study the effect of B401 treatment on HD symptoms at the early stages. In addition, we chose 3-month R6/2 HD mice to study the effect of B401 treatment on HD symptoms at the later stage. All protocols in the experiment were executed according to the international guidelines for care and use of laboratory animals.

\section{Behavior analysis in $\mathrm{R} 6 / 2$ mice}

For determining motor ability and coordination in R6/2 mice, a rotarod testing was used in this study. A standard rotarod (Ugo Basile North America Inc., Schwenksville, PA, USA) was applied on R6/2 mice and their wild type (WT). As described previously, R6/2 mice were acclimatized to the apparatus at the minimum speed of $4 \mathrm{rpm}$ for 20 seconds during each trial. ${ }^{18}$ Following acclimatization, the rod was progressively accelerated from $4 \mathrm{rpm}$ to $40 \mathrm{rpm}$ over a duration of 5 minutes. Latency to fall from the rod for R6/2 mice and their WT was recorded.

\section{Skin blood flow in $\mathrm{R} 6 / 2$ mice}

Laser Doppler imager (Moor Instruments, Axminster, UK) was put into practice to scan regional dermal microvascular blood flow in R6/2 mice without skin contact. ${ }^{19}$ Through the camera positioned at $18 \mathrm{~cm}$ above the skin, the blood flow would be recorded with the data acquisition software (MoorFLPI measurement software, Version 3.0; Moor Instruments) in arbitrary perfusion units.

\section{Brain morphology in $\mathrm{R} 6 / 2$ mice}

Following the methods previously described, R6/2 mice were imaged with magnetic resonance imaging (MRI) to study their brain size in cerebrum, midbrain, and cerebellum. ${ }^{20}$ Like rodents of MRI studies, R6/2 mice were anesthetized using 5\% isoflurane before they were positioned and fixed onto a plastic frame. While anesthetic was administered through a facemask, mice were in stable anesthesia, typically between $1 \%$ and $2 \%$ isoflurane for the duration of the MRI scanning. The $7 \mathrm{~T}$ horizontal bore MRI system (Bruker BioSpec 70/30 USR; Bruker BioSpin Corporation, Billerica, MA, USA) was used to form the image of brain according to three orthogonal planes: axial, sagittal, and coronal. With the aid of Vitrea Core software (Toshiba Medical Systems, Minnetonka, MN, USA), a 3D reconstruction of brain was created. Furthermore, the brain was first outlined in each axial image, primarily based on its anatomical location relative to adjacent organs, making it easier to calculate the volume of brain size. Eventually, terminal anesthesia with sodium pentobarbital $(50 \mathrm{mg} / \mathrm{kg}$ ) was given to mice at 3 months of age through intraperitoneal injection. After their vital signs disappear, their brains were removed, weighed, volume measured, and then fixed for staining.

\section{Brain immunohistochemical staining in $\mathrm{R} 6 / 2$ mice}

For immunohistochemical staining, anesthetized R6/2 HD and WT mice were cardiac perfused first with phosphatebuffered saline containing $4 \%$ formaldehyde and then fixed with $4 \%$ formaldehyde (EM grade). Brain specimens were then embedded in paraffin, cut into sections $(5 \mu \mathrm{m})$, and placed on coated slides. By using the heat-induced epitope retrieval method, sections were separately stained at room temperature for 1 hour with antibodies of mutant huntingtin (Abcam Inc., Cambridge, MA, USA), BDNF (Santa Cruz Biotechnology Inc., Santa Cruz, CA, USA), vascular endothelial growth factor (VEGF) (Cell Signaling Technology Inc., Danvers, MA, USA), and tumor necrosis factoralpha (TNF- $\alpha$ ) (Cell Signaling Technology Inc.). Detection was performed by incubation with biotinylated secondary antibodies (Novolink ${ }^{\mathrm{TM}}$ polymer detection system 1), for 30 minutes at room temperature, followed by a 30 -minute incubation with avidin-biotin-horseradish peroxidase (HRP) peroxidase complex (Novolink ${ }^{\mathrm{TM}}$ polymer detection system 1). Visualization was performed with DAB Chromogen (Novolink ${ }^{\mathrm{TM}}$ polymer detection system $\mathrm{l}$ ) and counterstaining with hematoxylin (Novolink ${ }^{\mathrm{TM}}$ polymer detection system 1) following supplier's protocol.

\section{Western blotting in the brain of R6/2 mice}

The removed brain tissue was kept in a buffer solution to maintain $\mathrm{pH}$ for the preparation of Western blot analysis. Thus, brain protein was subjected to sodium dodecyl sulfatepolyacrylamide gel electrophoresis, and transferred to polyvinylidene difluoride membrane. This study uses antibodies such as alpha-tubulin (Thermo Fisher Scientific Inc., Waltham, MA, USA), beta-actin (Thermo Fisher Scientific Inc.), mutant huntingtin (Abcam Inc.), BDNF (Santa Cruz Biotechnology Inc.), VEGF (Cell Signaling Technology Inc.), and TNF- $\alpha$ (Cell Signaling Technology Inc.). They were identified by means of a horseradish peroxidase-linked secondary antibody. In addition, utilizing ECL Western blotting detection reagents (GE Healthcare Life Sciences, Barrington, IL, USA) makes immunoreactive bands perceptible, and LAS-4000 (GE Healthcare Life Sciences) avails detection of the chemiluminescence. ImageJ software (version 1.48t; Wayne Rasband, Washington DC, USA) makes it possible to perform densitometric assessments of the bands. 


\section{Statistical analysis}

The data of cell viability assay were obtained from at least six independent experiments, each done in triplicate, while the data of Western blot analysis were obtained from at least six independent experiments. The values of all data were expressed as the means \pm standard error of the mean. Differences between groups were evaluated by one-way analysis of variance followed by Student-Newman-Keuls multiple comparisons posttest. The $P$-values of at least $<0.05$ were considered significant.

\section{Results}

\section{Effect of B40I treatment on glutamate- treated $\mathrm{SH}-\mathrm{SY} 5 \mathrm{Y}$ cells viability}

Effect of B401 treatment on glutamate-treated SH-SY5Y cells viability is shown in Figure 1B. As detected by MTT method, glutamate treatment in SH-SY5Y cells caused a decrease in cell viability. SH-SY5Y cell viability was reduced approximately $35 \%$ compared to untreated SH-SY5Y cells by exposure to $10 \mathrm{mM}$ glutamate. When the medium was cultured in the presence of MK-801, an NMDA receptor antagonist, SH-SY5Y cell viability was significantly increased compared to glutamate-treated SHSY5Y cells $(P<0.01)$. Consistent with the effect of MK-801, SH-SY5Y cell viability was significantly increased compared to glutamate-treated SH-SY5Y cells $(P<0.01-0.05)$ when the medium was cultured in the presence of different concentrations of the Chinese herbal formula B401 (10-30 mg/mL).

\section{Effect of oral B40I treatment on survival, body weight, and motor performance of $\mathrm{R} 6 / 2 \mathrm{HD}$ mice}

A total of 42 R6/2 HD mice (22 R6/2 HD mice with oral B401 treatment and 20 R6/2 HD mice with sham treatment) were observed in this study. We compared the life span and body weight of HD mice with and without oral B401 treatment. Our results showed that survival was prolonged and the loss of body weight was alleviated in R6/2 HD mice under B401 treatment (Figure 2). Survival duration of R6/2 HD mice with oral B401 treatment was longer than those with sham treatment (Figure 2A). R6/2 HD mice with sham treatment all died at the end of 17 weeks of age (Figure 2A, solid circles), while R6/2 HD mice with oral B401 treatment all died at the end of 22 weeks of age (Figure 2B, unfilled circles). Before 6 weeks of age, the R6/2 HD mice with oral B401 treatment exhibited a normal survival number and an increase in body weight similar to that observed in those R6/2 HD mice with sham treatment (Figure 2B). After 8 weeks of age, body weight of R6/2 HD mice with oral B401 treatment still increased (Figure 2B, solid circles), while body weight of R6/2 HD mice with sham treatment reduced than those R6/2 HD mice with oral B401 treatment (Figure $2 \mathrm{~B}, P<0.01-0.05$, unfilled circles vs solid circles). In the process of breeding animals, we also compared motor performance with and without oral B401 treatment. By using rotarod testing, we observed that the latencies to fall of R6/2 HD mice with oral B401 treatment were significantly longer than those with sham treatment from 8 weeks of age and thereafter (Figure 2C, $P<0.01-0.05$ ).

\section{Effect of intraperitoneal B40 I injection on subcutaneous microcirculation of R6/2 HD mice}

The subcutaneous microcirculatory flows from dorsal and ventral aspects in R6/2 HD mice with and without intraperitoneal B401 injection are compared in Figure 3. By using moorFLPI high-resolution laser Doppler imager, we observed
A

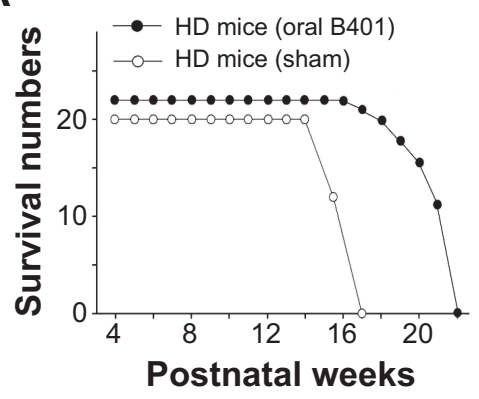

B

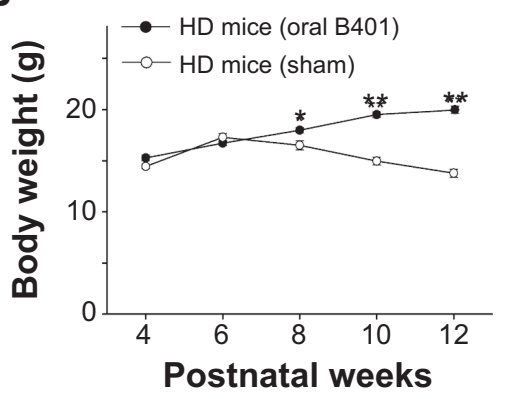

C

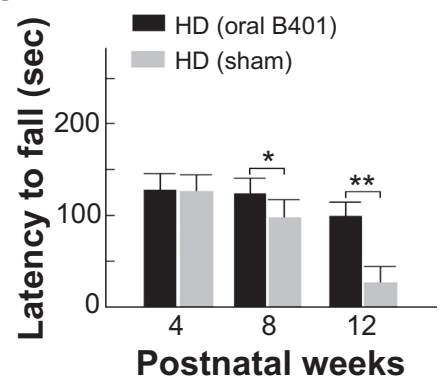

Figure 2 Survival, loss of body weight, and motor function of R6/2 HD mice were improved under oral B40I treatment.

Notes: (A) Survival duration of R6/2 HD mice under oral B40I treatment was increased than those with sham treatment. (B) Body weight of R6/2 HD mice under oral B40I treatment (HD mice oral B40I, $n=22$ ) was significantly greater than those with sham treatment (HD mice sham, $n=20$ ) from 8 weeks of age and thereafter. (C) Latencies to fall of $\mathrm{R} 6 / 2 \mathrm{HD}$ mice under oral B40I treatment (HD oral B40I, $n=16$ ) were significantly increased than those with sham treatment (HD sham, $n=15)$ from 8 weeks of age and thereafter as observed by rotarod testing. Values are mean $\pm \mathrm{SEM}(* * P<0.01$, $* P<0.05$, one-way ANOVA followed by a Student-Newman-Keuls multiple comparisons posttest).

Abbreviations: HD, Huntington's disease; SEM, standard error of the mean; ANOVA, analysis of variance. 

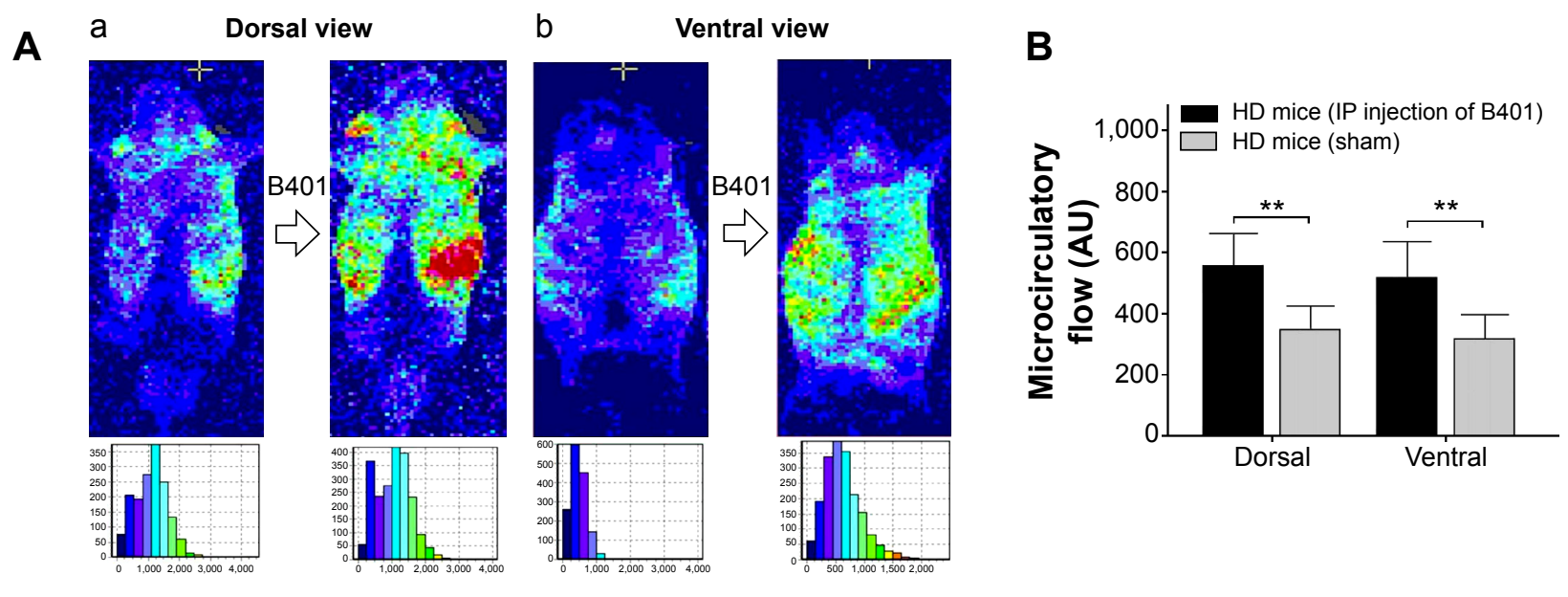

Figure 3 Subcutaneous microcirculation of 3-month R6/2 mice was enhanced under IP B40I injection.

Notes: (A) Dorsal (a) and ventral (b) subcutaneous microcirculatory flow imaging before and after intraperitoneal B40I (I00 mg/kg) injection by using moorFLPI laser Doppler imager. (B) Dorsal and ventral subcutaneous microcirculatory flows of 3-month R6/2 mice with intraperitoneal B40I injection (HD mice IP injection of B40I, $n=6$ ) were significantly increased than those with sham treatment (HD mice sham, $n=6$ ). Values are mean \pm SEM ( $* * P<0.0$ I, one-way ANOVA followed by a Student-NewmanKeuls multiple comparisons posttest).

Abbreviations: AU, arbitrary perfusion units; HD, Huntington's disease; IP, intraperitoneal; SEM, standard error of the mean; ANOVA, analysis of variance.

that dorsal and ventral subcutaneous microcirculatory flows of 3-month R6/2 HD mice with intraperitoneal B401 injection were increased than those with sham treatment (Figure 3A). Then, we measured and compared subcutaneous microcirculatory flows of the dorsal and ventral aspects in 3-month R6/2 HD mice. Our data showed that both subcutaneous microcirculatory flows of the dorsal and ventral aspects of 3-month R6/2 HD mice with intraperitoneal B401 injection were significantly greater than those R6/2 HD mice with sham treatment (Figure 3B, $P<0.01$ ).

\section{Effect of B40I treatment on brain atrophy of R6/2 HD mice}

Concomitant to behavioral assessments, MRI was used to noninvasively study brain structure. We observed visible atrophy in the brain of 3-month R6/2 mice (Figure 4A). The MRI data revealed that $\mathrm{R} 6 / 2$ mice develop progressive, region-specific, and age-dependent brain atrophy, while the Chinese herbal formula B401 might alleviate brain atrophy in R6/2 mice. Interestingly, we also observed that volume and weight changes of cerebrum in the brain of 3-month R6/2 mice were significantly correlated with changes in midbrain and cerebellum but not correlated with changes in the brainstem (Figure 4B). In addition, brain atrophy of 3-month R6/2 mice was alleviated under oral B401 treatment because both volume and weight of cerebrum, midbrain, and cerebellum in 3-month R6/2 HD mice with oral B401 treatment were significantly greater than those R6/2 HD mice with sham treatment (Figure 4B, $P<0.05$ ).

\section{Effect of oral B40I treatment on mutant huntingtin aggregation in the brain of R6/2 HD mice}

The deposition of mutant huntingtin is a neuropathological hallmark of HD. ${ }^{21}$ We have evaluated the levels of the mutant huntingtin in cerebral cortex, striatum, hippocampus, and medulla from WT littermates and 2-month R6/2 mice treated with vehicle or B401. We aimed at determining whether mutant huntingtin level in the brain was reduced after oral treatment with B401 in 2-month R6/2 HD mice. As shown in Figure 5A, our immunohistochemical staining study revealed that the expression levels of mutant huntingtin in the cortex, striatum, and hippocampus were visibly increased in saline-treated R6/2 HD mice (HD, sham) compared to the WT, and that oral B401 treatment visibly reduces mutant huntingtin levels in the cortex, striatum, and hippocampus of R6/2 HD mice (HD, B401). Moreover, we observed increased levels of mutant huntingtin not only in the striatal neurons but also in the cortical neurons of saline-treated R6/2 HD mice (Figure 5A). The results were confirmed by Western blotting (Figure 5B). We observed that expression levels of mutant huntingtin in whole brain tissue of saline-treated R6/2 HD mice were significantly increased compared to their WT (Figure 5B, HD sham, $P<0.01$ vs WT), while expression levels of mutant huntingtin in brain tissue were significantly reduced in R6/2 HD mice under oral B401 treatment (Figure 5B, HD sham, $P<0.01$ vs HD B401). 

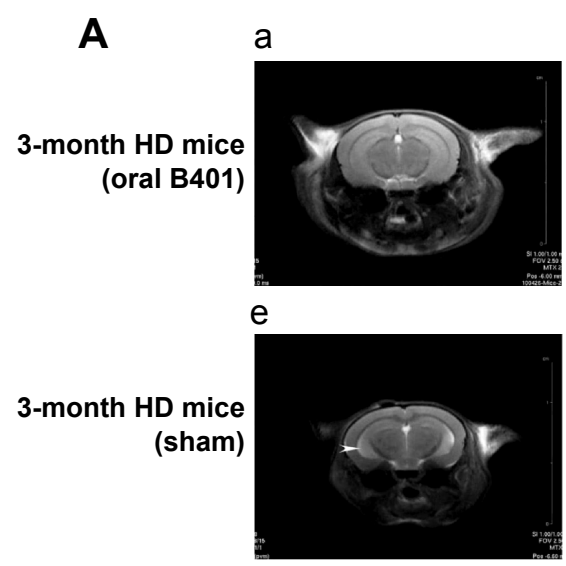

B

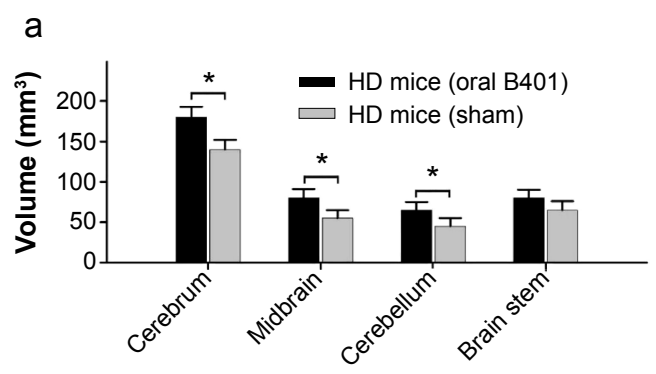

b

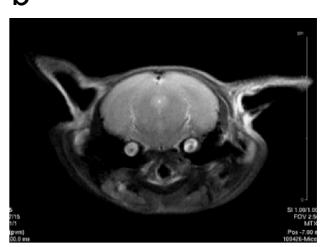

f

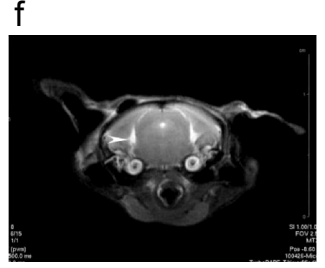

C

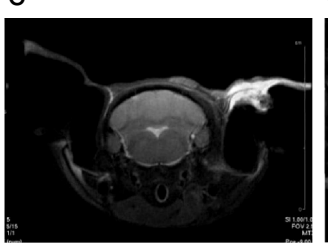

g

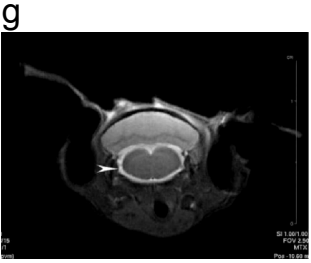

d

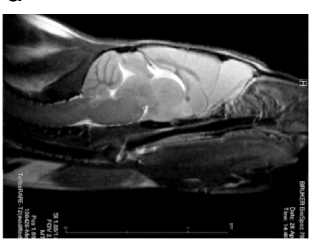

h

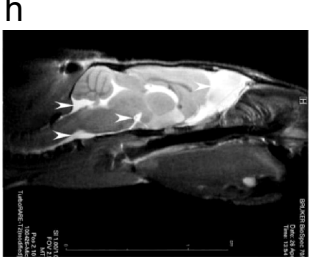

b

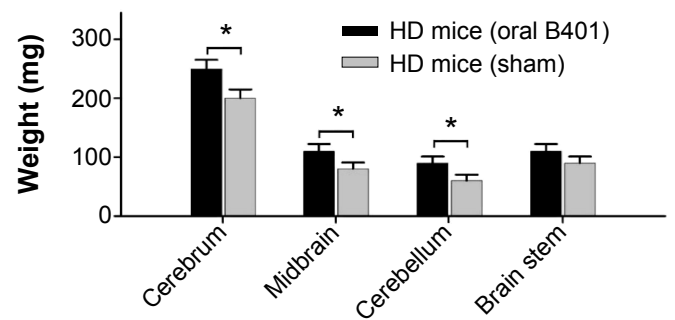

Figure 4 Brain atrophy of 3-month R6/2 mice was alleviated under oral B40I treatment.

Notes: (A) Magnetic resonance images corresponding to the brain at different cross- and longitudinal-section in 3-month R6/2 mice with oral B40I treatment (a-d) is less than those with sham treatment (e-h). (B) The volume (a) and weight (b) of the cerebrum, midbrain, and cerebellum in 3-month R6/2 mice with oral B40I treatment ( $\mathrm{HD}$ mice oral $\mathrm{B} 40 \mathrm{I}, \mathrm{n}=6$ ) are significantly greater than those with sham treatment (HD mice sham, $n=6$ ). Values are mean $\pm S E M(* P<0.05$, one-way $A N O V A$ followed by a Student-Newman-Keuls multiple comparisons posttest).

Abbreviations: HD, Huntington's disease; SEM, standard error of the mean; ANOVA, analysis of variance.

\section{Effect of oral B40I treatment on BDNF and VEGF expressions in the brain of R6/2 HD mice}

The neurotrophic hypothesis of HD proposes that impaired production of BDNF, a neurotrophin of growth factors, contributes to degeneration of neurons in the striatum. ${ }^{22}$ We have evaluated the expression levels of the BDNF in cerebral cortex, striatum, hippocampus, and medulla from WT and R6/2 HD mice treated with vehicle or B401. As shown in Figure 6A, our immunohistochemical staining study revealed that the BDNF expression levels in the cortex, striatum, and hippocampus of sham-treated R6/2 HD mice (HD, sham) were visibly decreased than their WT but were visibly increased in R6/2 mice under oral B401 treatment (HD, B401). We quantified the expression levels of BDNF by Western blotting analysis as shown in Figure 6B. The expression levels of BDNF in the brain tissue of 2-month R6/2 mice were significantly weaker than those of their WT (Figure 6B, HD sham, $P<0.01$ vs WT) but were significantly enhanced in the brain tissue of $\mathrm{R} 6 / 2$ mice under oral B401 treatment (Figure 6B, HD B401, $P<0.01$ vs HD sham).
In the brain, VEGF also plays a crucial role in neuroprotective and angiogenic effects. ${ }^{23}$ From immunohistochemical staining, we found that oral B401 treatment visibly enhanced expressions of VEGF in the cortex, striatum, and hippocampus of 2-month R6/2 mice (Figure 7A). By Western blotting analysis, we found that the expression levels of VEGF in the brain tissue of 2-month R6/2 mice were significantly weaker than those of their WT (Figure 7B, HD sham, $P<0.01$ vs WT) but were significantly enhanced in the brain tissue of R6/2 mice under oral B401 treatment (Figure 7B, HD sham, $P<0.01$ vs HD B401).

\section{Effect of oral B40I treatment on TNF- $\alpha$ expression in the brain of R6/2 HD mice}

As suggested in previous studies, inflammation is believed to be intimately involved in the pathogenesis and progression of neurologic diseases. ${ }^{24}$ Thus, we examined whether the Chinese herbal formula B401 alleviates inflammation in the brain of R6/2 mice. In this study, TNF- $\alpha$ is a key marker for inflammation. ${ }^{25}$ By immunohistochemical staining, we observed that the expressions of TNF- $\alpha$ in the cortex, striatum, and hippocampus of 2-month R6/2 mice were reduced under oral B401 treatment (Figure 8A). By Western blotting 
A

Cortex

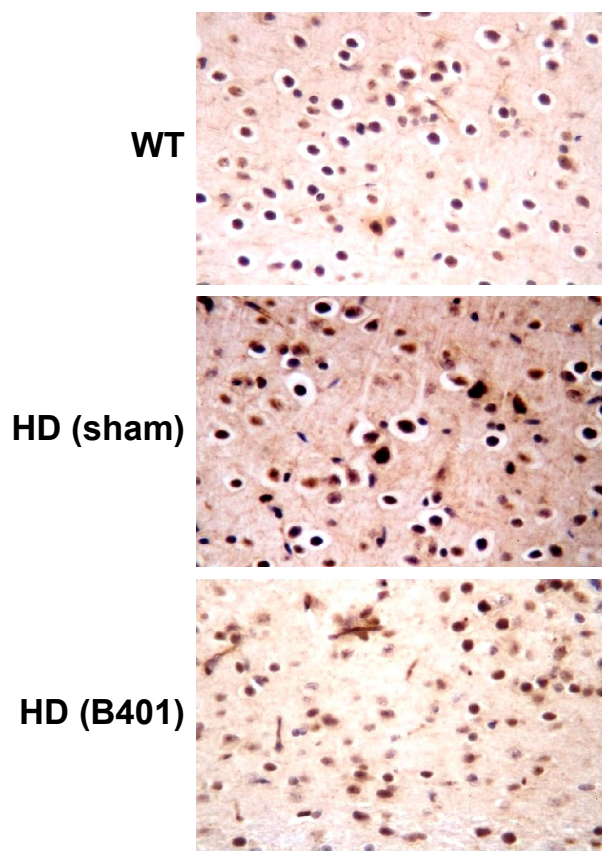

B a

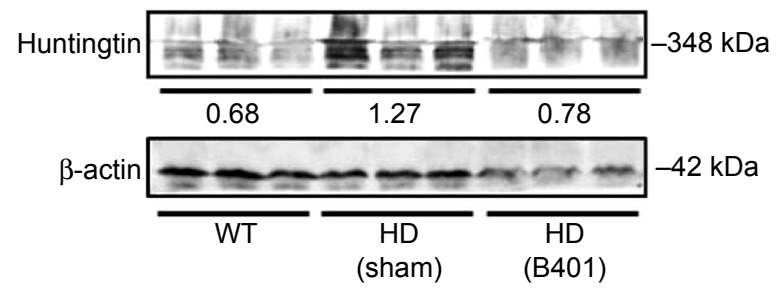

Striatum

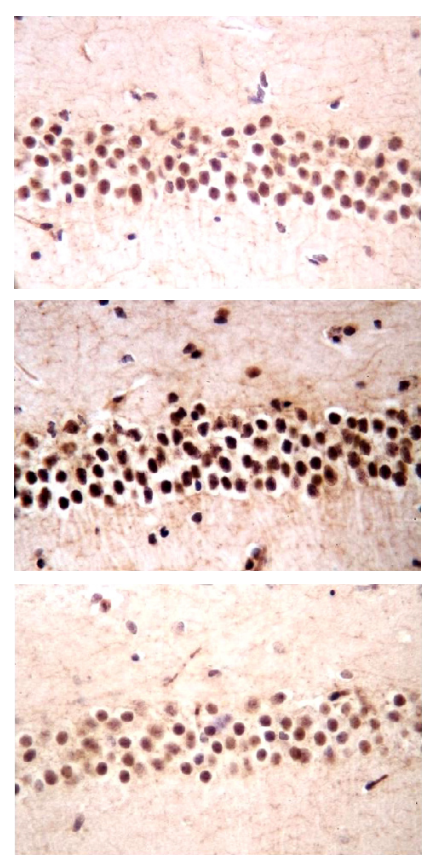

Hippocampus

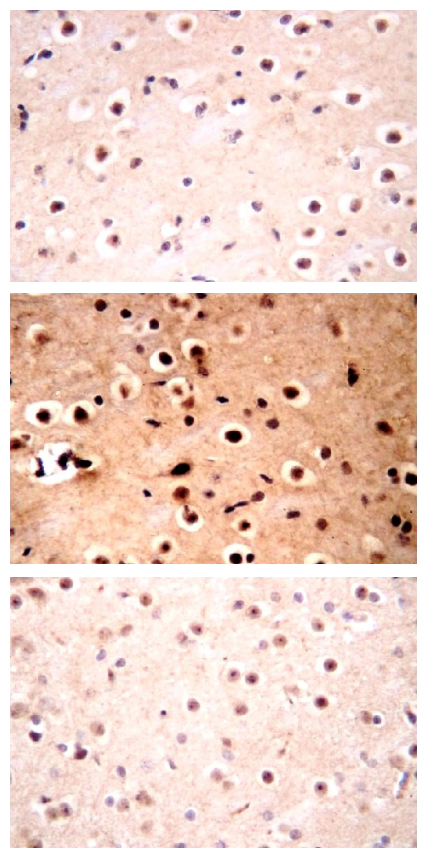

b

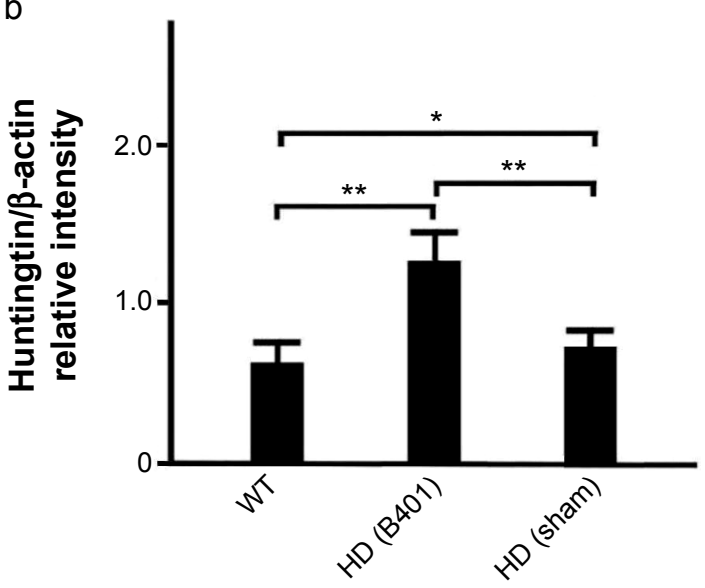

Figure 5 Mutant huntingtin aggregation in the brain tissue of 2-month R6/2 mice was downregulated under oral B40I treatment.

Notes: (A) Immunohistochemical staining illustrates the expression levels of mutant huntingtin in the cortex, striatum, and hippocampus of 2-month R6/2 mice and their WT. Expression levels of mutant huntingtin in the brain tissue of 2-month R6/2 mice were visibly reduced under oral B40I treatment. The width $\times$ height of each figure is $150 \mu \mathrm{m} \times 100 \mu \mathrm{m}$. (B) Western blotting analysis shows the following: (a) expression levels of aggregation of mutant huntingtin in whole brain tissue of 2-month R6/2 mice with oral B40I and sham treatments, and their WT and (b) quantified huntingtin levels in brain tissue of 2-month R6/2 mice (HD sham, n=6) were significantly increased than their WT $(n=6)$, and were significantly reduced under oral B40I treatment (HD B40I, $n=6)$. Values are mean $\pm S E M(* P<0.05$, $* * P<0.01$, one-way ANOVA followed by a Student-Newman-Keuls multiple comparisons posttest).

Abbreviations: WT, wild type; HD, Huntington's disease; SEM, standard error of the mean; ANOVA, analysis of variance.

analysis, we found that the expression levels of TNF- $\alpha$ in the brain tissue of 2-month R6/2 mice were significantly increased than those of their WT (Figure 8B, HD sham, $P<0.01$ vs WT) but were significantly reduced in the brain tissue of R6/2 mice under oral B401 treatment (Figure 8B, HD B401, $P<0.01$ vs HD sham).

\section{Discussion}

In this study, we evaluated the effects of the Chinese herbal formula B401 in glutamate-treated SH-SY5Y cells in vitro and R6/2 HD mouse model in vivo. In the in vitro experiments, we found that glutamate-treated SH-SY5Y cells viability was increased under pretreatment with the Chinese herbal formula B401 (Figure 1B). In the in vivo experiments, we found that life span was increased (Figure 2A), body weight loss was alleviated (Figure 2B), motor ability was enhanced (Figure 2C), and brain atrophy was alleviated (Figure 4) in R6/2 HD mice under oral B401 treatment. Subcutaneous microcirculatory blood flow was improved under intraperitoneal B401 injection (Figure 3). 


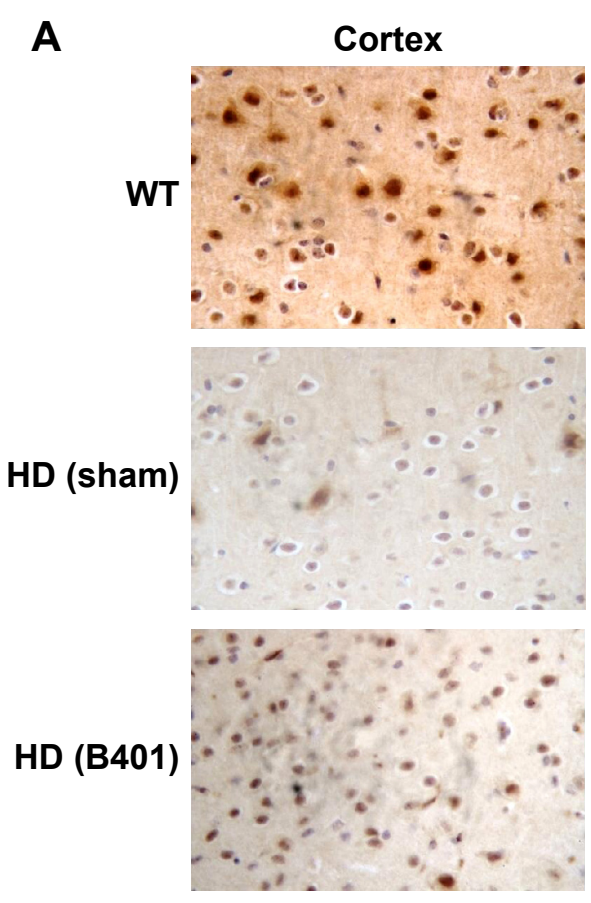

B a
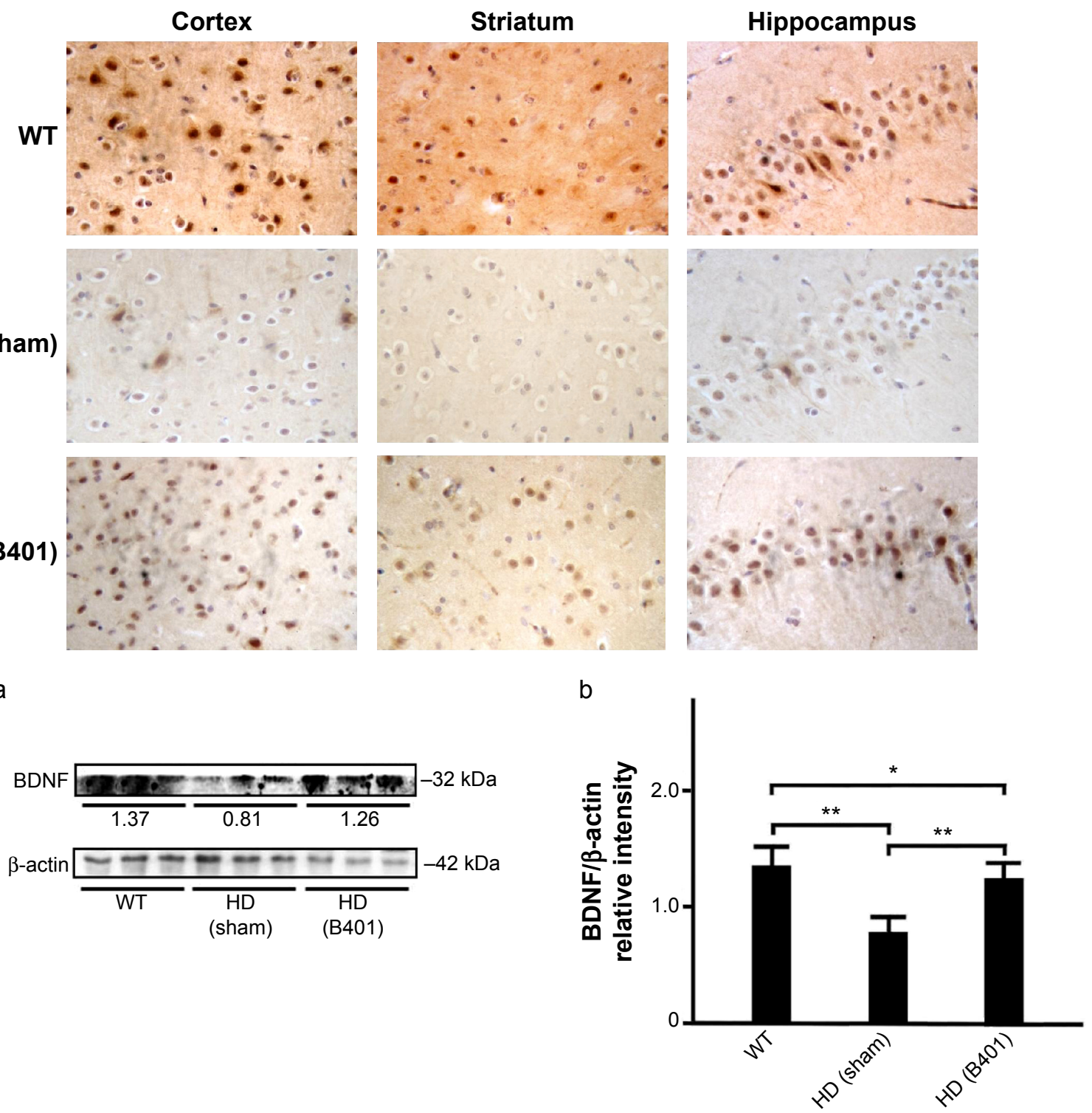

Figure 6 BDNF levels in the brain tissue of 2-month R6/2 mice were upregulated under oral B40I treatment.

Notes: (A) Immunohistochemical staining illustrates expression levels of BDNF in the cortex, striatum, and hippocampus of 2-month R6/2 mice and their WT. Expression levels of BDNF in the brain tissue of 2-month R6/2 mice were visibly enhanced under oral B40I treatment. The width $\times$ height of each figure is I50 $\mu \mathrm{m} \times 100 \mu \mathrm{m}$. (B) Western blotting analysis shows the following: (a) expression levels of BDNF in whole brain tissue of 2-month R6/2 mice and their WT and (b) quantified brain BDNF levels of 2-month $R 6 / 2$ mice (HD sham, $n=6$ ) were significantly reduced than their WT $(n=6)$, and were significantly increased under oral B40I treatment (HD B40I, $n=6)$. Values are mean \pm SEM $(* P<0.05, * * P<0.0$ I, one-way ANOVA followed by a Student-Newman-Keuls multiple comparisons posttest).

Abbreviations: WT, wild type; HD, Huntington's disease; BDNF, brain-derived neurotrophic factor; SEM, standard error of the mean; ANOVA, analysis of variance.

Further immunohistochemical staining and Western blotting analysis show that mutant huntingtin aggregation and TNF- $\alpha$ expression, a marker of inflammation, were reduced in $\mathrm{R} 6 / 2$ HD mice under oral B401 treatment (Figures 5 and 8). In addition, BDNF and VEGF expressions were enhanced in brain tissue of R6/2 HD mice under oral B401 treatment (Figures 6 and 7).

The chromatographic fingerprint analysis of the Chinese herbal formula B401 is demonstrated in Figure 1A. The marker compounds of the Chinese herbal formula B401 are ginsenosides from $P$. ginseng, oleanolic acid from $L$. fructus, formononetin from A. membranaceus, 5-hydroxymethylfurfural from R. glutinosa, ferulic acid from L. fructus, and wedelolactone from Eclipta prostrata. As for traditional Chinese medicine, to understand the limited value of these single herbs is necessary for us. We must know that none of these herbs have much therapeutic value. If we use any of them alone, we may encounter problems. That is why traditional Chinese medicine is all about using herbs together. Many synthetic drugs have poor patient compliance because 
A

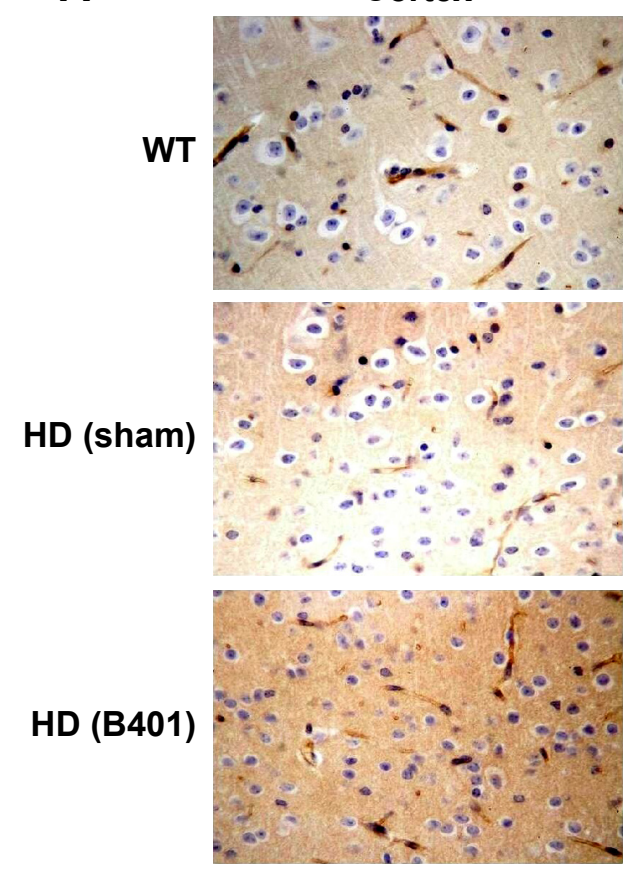

B a
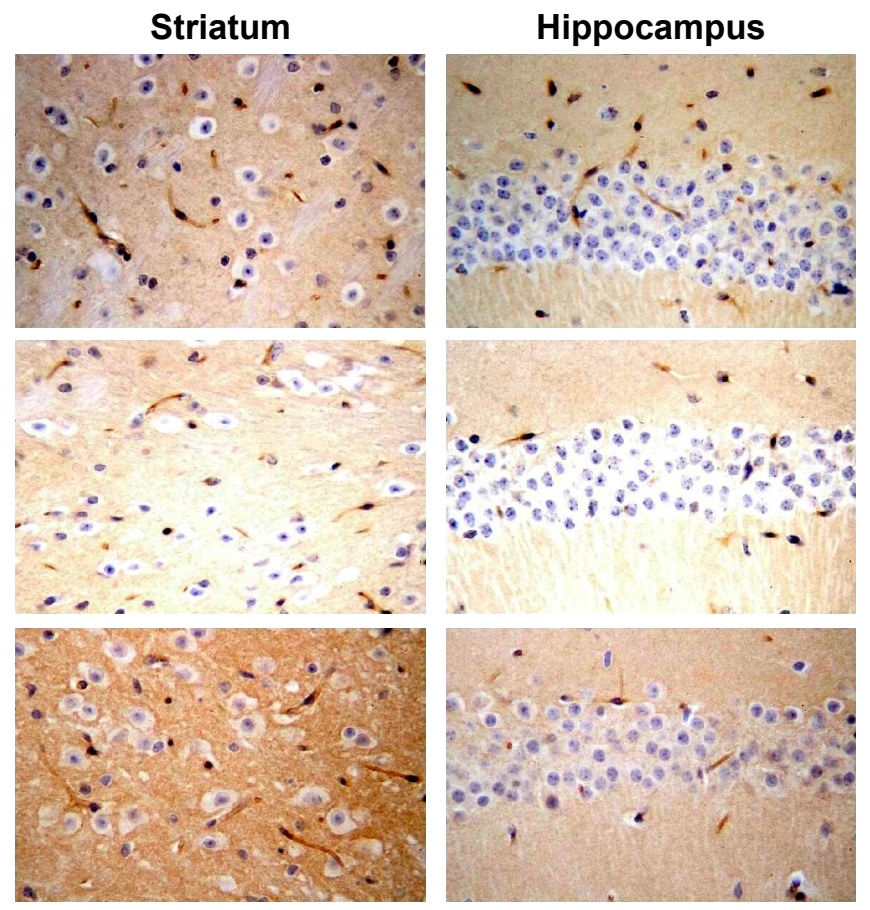

b

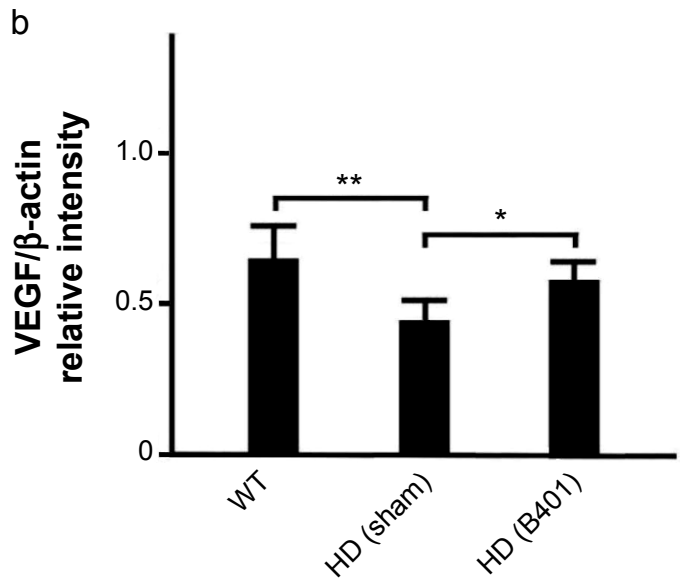

Figure 7 VEGF levels in the brain tissue of 2-month R6/2 mice were upregulated under oral B40I treatment.

Notes: (A) Immunohistochemical staining illustrates expression levels of VEGF in the cortex, striatum, and hippocampus of 2-month R6/2 mice and their WT. Expression levels of VEGF in the brain tissue of 2-month R6/2 mice were visibly enhanced under oral B40I treatment. The width $\times$ height of each figure is I50 $\mu \mathrm{m} \times 100 \mu \mathrm{m}$. (B) Western blotting analysis shows the following: (a) expression levels of VEGF in whole brain tissue of 2-month R6/2 mice and their WT and (b) quantified brain VEGF levels of 2-month $\mathrm{R} 6 / 2$ mice (HD sham, $n=6$ ) were significantly reduced than their WT $(n=6)$, and were significantly increased under oral B40I treatment (HD B40I, $n=6)$. Values are mean \pm SEM $(* P<0.05, * * P<0.0$ I, one-way ANOVA followed by a Student-Newman-Keuls multiple comparisons posttest).

Abbreviations: WT, wild type; HD, Huntington's disease; VEGF, vascular endothelial growth factor; SEM, standard error of the mean; ANOVA, analysis of variance.

of many unwanted but unavoidable side effects. Therefore, herbal treatment is being preferred over conventional treatments. As suggested in previous studies, there is much attention toward herbal remedy of many brain disorders such as $\mathrm{AD}, \mathrm{PD}$, and HD. ${ }^{11-13}$

Mutant huntingtin inclusions correlate with the HD progression in a mouse model. ${ }^{23}$ In the same study, it is shown that the reduction of huntingtin aggregation may mitigate behavioral and cognitive deficits. Therefore, therapeutic that reduces huntingtin aggregate formation or inactivates mutant huntingtin protein might be beneficial for HD progression. Our immunohistochemical evidence showed that expression levels of mutant huntingtin aggregation in the cortex, striatum, and hippocampus of R6/2 HD mice were obviously weaker than their WT (Figure 5A). Also, evidence from Western blotting analysis showed that expression levels of mutant huntingtin in the brain of R6/2 HD mice were significantly increased than their WT (Figure 5B). Expression levels of mutant huntingtin were significantly reduced in the brain tissue of R6/2 HD mice under oral B401 treatment. 
A

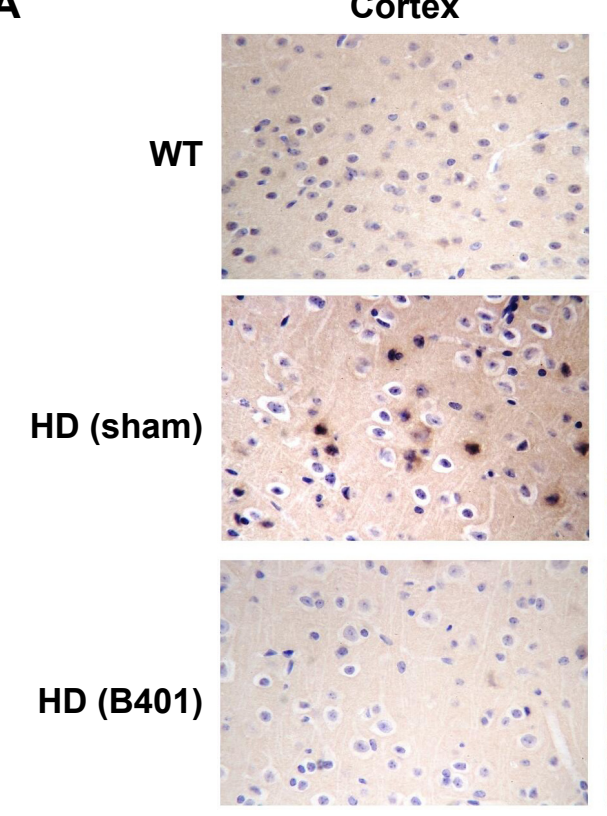

Striatum

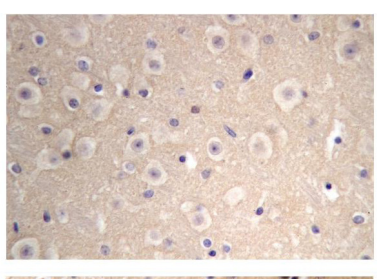

Hippocampus

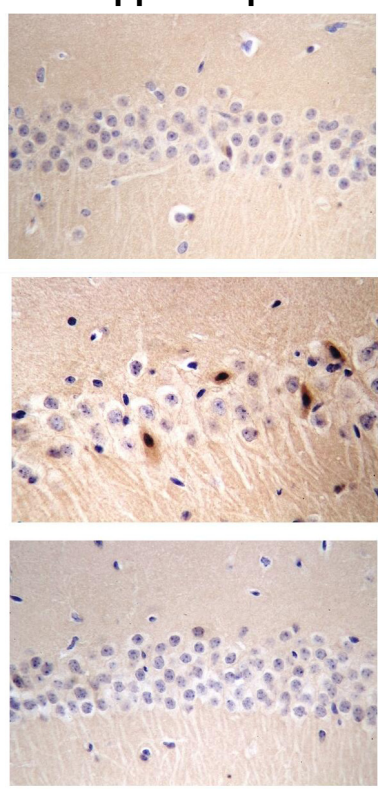

B

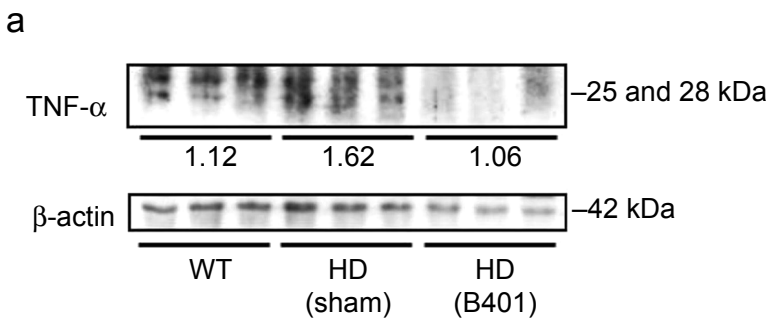

b

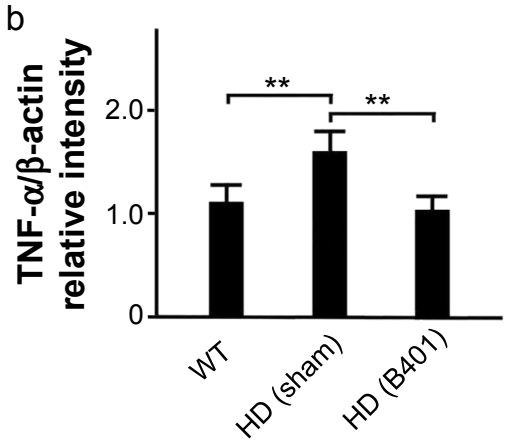

Figure 8 TNF- $\alpha$ levels in the brain tissue of 2-month R6/2 mice were downregulated under oral B40I treatment.

Notes: (A) Immunohistochemical staining illustrates expression levels of TNF- $\alpha$ in the cortex, striatum, and hippocampus of 2-month R6/2 mice and their WT. Expression levels of TNF- $\alpha$ in the brain tissue of 2-month R6/2 mice were visibly reduced under oral B40I treatment. (B) Western blotting analysis shows the following: (a) expression levels of aggregation of TNF- $\alpha$ in whole brain tissue of 2-month R6/2 mice with oral B40I and sham treatments, and their WT and (b) quantified brain TNF- $\alpha$ levels of 2-month R6/2 mice (HD sham, $n=6$ ) were significantly increased than their WT $(n=6)$, and significantly reduced under oral B40I treatment (HD B40I, $n=6)$. Values are mean \pm SEM (**P $<0.0$ I, one-way ANOVA followed by a Student-Newman-Keuls multiple comparisons posttest).

Abbreviations: WT, wild type; HD, Huntington's disease; TNF- $\alpha$, tumor necrosis factor-alpha; SEM, standard error of the mean; ANOVA, analysis of variance.

It is therefore possible that Chinese herbal formula B401 could reduce mutant huntingtin aggregation and promote the clearance of huntingtin toxic fragments.

Several evidences have indicated that excitotoxicity contributes to neuronal death in HD. ${ }^{26,27}$ Electrophysiological evidence documented increased glutamate release from cortical afferents in HD mice and increased excitotoxicity. ${ }^{28}$ The evidence described above documents that huntingtin aggregation may increase NMDA receptor sensitivity to NMDA and enhance NMDA-evoked currents in brain of HD mice. Thus, cortical hyperexcitability might be a major pathology in HD. Another neurochemical evidence suggests that the deficit of connectivity between the cortex and striatum may play an important role in the excitotoxic process and contribute to the HD phenotype..$^{29,30}$ The MK- 801 is an NMDA receptor antagonist that has effective neuroprotection in excitotoxic neurodegeneration. ${ }^{31,32}$ In this study, we evaluated the effect of B401 on neurotoxicity in glutamate-treated SH-SY5Y neuroblastoma cell lines. Our results demonstrated that glutamate-induced neurotoxicity in SH-SY5Y cells was reduced under pretreatment with MK-801 and the Chinese herbal formula B401 individually (Figure 1B).

In the later stages, HD is characterized by motor dysfunction, progressive dementia, and memory loss. ${ }^{33}$ Lifethreatening complications may result from injuries related to serious falls, poor circulation, infection, and inflammation. We further take advantage of the Chinese herbal formula B401 to test potential therapeutic treatment strategies of R6/2 
HD mice. Multiple parameters likely govern life span of R6/2 HD mice including peripheral effects such as weight loss and muscle atrophy, and central effects such as brain atrophy. In this study, we observed that the body weight of R6/2 HD mice was significantly reduced from 2 months and thereafter, while R6/2 HD mice under oral B401 treatment still kept normal body weight until 3 months of age (Figure 2B). In addition to benefits in life span and body weight, motor function of R6/2 HD mice under oral B401 treatment was improved as observed by rotarod testing (Figure 2C). Similar to our observation, it has been reported that some traditional Chinese herbs may relieve some of the HD symptoms such as rigidity and dystonia. ${ }^{13}$

The pathology of HD brain is notably specific with prominent cell loss and atrophy in the caudate and putamen. ${ }^{34}$ In the later stages, HD causes atrophy of the striatum and up to 95\% neuronal loss. ${ }^{35}$ By using MRI morphometric analysis, we observed that the volume and weight of cerebrum, midbrain, and cerebellum of 3-month R6/2 mice were significantly decreased than their WT. Consistent with our study, previously neurologists have confirmed that HD patients had significant volume reductions in almost all brain structures, specifically in the cortex. ${ }^{36}$ In this study, we also observed that brain atrophy of 3-month R6/2 mice was significantly alleviated under oral B401 treatment (Figure 4, $P<0.05$ ). As suggested from previous study, BDNF in the cerebral cortex plays a role in striatal and cortical vulnerability of HD. ${ }^{37}$ Mutant huntingtin may affect BDNF transcription by regulating the activity of cAMP response element-binding protein (CREB), the BDNF promoter. ${ }^{38}$ In addition, BDNF treatment may delay motor impairment in R6/2 HD mice because these mice exhibited a significant slowing in latency to fall from rotarod test. ${ }^{37}$ In our study, expressions of BDNF in the cortex, striatum, and hippocampus of R6/2 HD mice were obviously weaker than their WT (Figure 6) but significantly enhanced in R6/2 HD mice under oral B401 treatment. It is possible that neuroprotection of Chinese herbal formula
B401 in HD brain might be via reducing mutant huntingtin aggregation and enhancing brain BDNF expression.

Besides neurological disease, cardiac failure may induce death in HD patients. ${ }^{39,40}$ In this study, we observed that subcutaneous microcirculatory flows were very poor in 3-month R6/2 HD mice (Figure 3A). The Chinese herbal formula B401 contains ingredients that could increase the quantity and stimulate the flow of breath and blood. By using moorFLPI high-resolution laser Doppler imager, we observed that dorsal and ventral subcutaneous blood flows of R6/2 HD mice were enhanced under intraperitoneal B401 injection (Figure 4A). In addition, we found that VEGF expressions in brain tissue of R6/2 HD mice were significantly increased under oral B401 treatment (Figure 7). The overexpression of VEGF may be used for the treatment of myocardial and peripheral ischemia, ${ }^{41,42}$ and for the treatment of AD and PD. ${ }^{43,44}$ Similarly, it is possible that neuroprotection of Chinese herbal formula B401 in HD brain might be via enhancing VEGF expression and strengthening cardiovascular function.

It has been reported that postmortem human HD tissue has a distinct profile of inflammatory mediators. ${ }^{45}$ In the study, it was observed that TNF- $\alpha$ was upregulated in the striatum of HD patients. Thus, the inflammatory mediators detected in the striatum might be a sign of the HD pathology. Evidence from our immunohistochemical and Western blotting analysis showed that expression levels of TNF- $\alpha$ in the brain of R6/2 HD mice were significantly increased than their WT but were significantly decreased in R6/2 HD mice under oral B401 treatment (Figure 8). Thus, we suggested that the Chinese herbal formula B401 might be a valuable therapeutic approach for HD medical treatment via inhibiting neuroinflammation.

\section{Conclusion}

$\mathrm{HD}$ is characterized by slow and progressive loss of neurons in the striatum of the central nervous system. As summarized in Figure 9, we have suggested that B401 treatment could downregulate mutant huntingtin aggregation in the brain

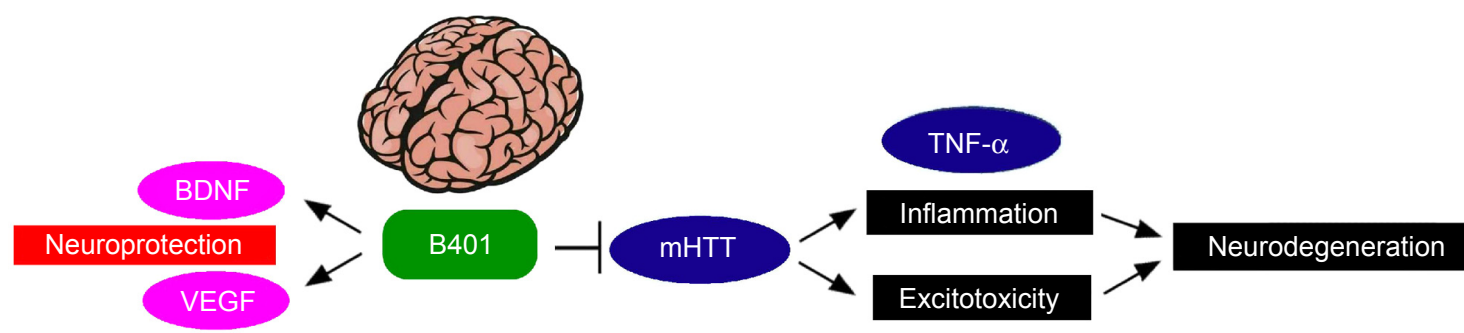

Figure 9 Schematic diagram illustrates that R6/2 HD mice under B40I treatment may improve neuroprotection via enhancing brain BDNF and VEGF gene expressions, and alleviate neurodegeneration via reducing mutant huntingtin (mHTT) aggregation, TNF- $\alpha$ gene expression (a marker of inflammation), and glutamate-induced excitotoxicity in the brain.

Abbreviations: BDNF, brain-derived neurotrophic factor; VEGF, vascular endothelial growth factor; TNF- $\alpha$, tumor necrosis factor-alpha; HD, Huntington's disease. 
of R6/2 HD mice. Downregulation of mutant huntingtin aggregation may reduce excitotoxic neurodegeneration and alleviate inflammation via reducing TNF- $\alpha$ expression in the brain of R6/2 HD mice. On the other hand, B401 treatment could protect brain via enhancing BDNF and VEGF expression in the brain of R6/2 HD mice. To sum up our study, we suggest that the herbal formula B401 can be used as a potential medical supplement in ameliorating neurodegenerative diseases of HD.

\section{Acknowledgments}

We acknowledge the Top University Project of NTNU, NTNU Transnational Research Centers Grant (103T3040B04), and Industry-University Cooperative Grant by Brion Research Institute of Taiwan. The funding agency had no role in the study design, data collection and analysis, and the decision to publish the manuscript.

\section{Disclosure}

The authors declare no conflicts of interest in this work.

\section{References}

1. Martin JB, Gusella JF. Huntington's disease. Pathogenesis and management. N Engl J Med. 1986;315:1267-1276.

2. The Huntington's Disease Collaboration Group. A novel gene containing a trinucleotide repeat that is expanded and unstable on Huntington's disease chromosomes. Cell. 1993;26:971-983.

3. Rigamonti D, Bauer JH, De-Fraja C, et al. Wildtype Huntingtin protects from apoptosis upstream of caspase-3. J Neurosci. 2000;20: 3705-3713.

4. Rigamonti D, Sipione S, Goffredo D, Zuccato C, Fossale E, Cattaneo E. Huntingtin's neuroprotective activity occurs via inhibition of procaspase-9 processing. J Biol Chem. 2001;276:14545-14548.

5. Gervais FG, Singaraja R, Xanthoudakis S, et al. Recruitment and activation of caspase- 8 by the Huntingtin-interacting protein Hip-1 and a novel partner Hippi. Nat Cell Biol. 2002;4:95-105.

6. Leavitt BR, Guttman JA, Hodgson JG, et al. Wild-type Huntingtin reduces the cellular toxicity of mutant Huntingtin in vivo. Am J Hum Genet. 2001;68:313-324.

7. Ho LW, Brown R, Maxwell M, Wyttenbach A, Rubinsztein DC. Wild type Huntingtin reduces the cellular toxicity of mutant Huntingtin in mammalian cell models of Huntington's disease. J Med Genet. 2001;38:450-452.

8. Zoghbi HY, Orr HT. Glutamine repeats and neurodegeneration. Annu Rev Neurosci. 2000;23:217-247.

9. Davies SW, Turmaine M, Cozens BA, et al. Formation of neuronal intranuclear inclusions underlies the neurological dysfunction in mice transgenic for the HD mutation. Cell. 1997;90:537-548.

10. Schilling G, Becher MW, Sharp AH, et al. Intranuclear inclusions and neuritic aggregates in transgenic mice expressing a mutant $\mathrm{N}$-terminal fragment of Huntingtin. Hum Mol Genet. 1999;8:397-407.

11. Dos Santos-Neto LL, de Vilhena Toledo MA, Medeiros-Souza P, de Souza GA. The use of herbal medicine in Alzheimer's disease - a systematic review. Evid Based Complement Alternat Med. 2006;3(4):441-445.

12. More SV, Kumar H, Kang SM, Song SY, Lee K, Choi DK. Advances in neuroprotective ingredients of medicinal herbs by using cellular and animal models of Parkinson's disease. Evid Based Complement Alternat Med. 2013;2013:957875.
13. Satoh T, Takahashi T, Iwasaki K, et al. Traditional Chinese medicine on four patients with Huntington's disease. Mov Disord. 2009;24(3): 453-455.

14. Mangiarini L, Sathasivam K, Mahal A, Mott R, Seller M, Bates GP. Instability of highly expanded CAG repeats in mice transgenic for the Huntington's disease mutation. Nat Genet. 1997;15:197-200.

15. Mangiarini L, Sathasivam K, Seller M, et al. Exon 1 of the HD gene with an expanded CAG repeat is sufficient to cause a progressive neurological phenotype in transgenic mice. Cell. 1996;87:493-506.

16. Stack EC, Kubilus JK, Smith K, et al. Chronology of behavioral symptoms and neuropathological sequela in R6/2 Huntington's disease transgenic mice. J Comp Neurol. 2005;490:354-370.

17. Wu CH, Lai XJ, Cheng CJ, Yu YC, Chang CY. Novel applications of digital holographic microscopy in therapeutic evaluation of Chinese herbal medicines. Appl Opt. 2014;53:27.

18. Rattray I, Smith E, Gale R, Matsumoto K, Bates GP, Modo M. Correlations of behavioral deficits with brain pathology assessed through longitudinal MRI and histopathology in the R6/2 mouse model of HD. PLoS One. 2013;8(4):e60012.

19. Kubli S, Waeber B, Dalle-Ave A, Feihl F. Reproducibility of laser Doppler imaging of skin blood flow as a tool to assess endothelial function. J Cardiovasc Pharmacol. 2000;6:640-648.

20. Cepeda-Prado E, Popp S, Khan U, et al. R6/2 Huntington's disease mice develop early and progressive abnormal brain metabolism and seizures. J Neurosci. 2012;32:6456-6467.

21. Gutekunst CA, Levey AI, Heilman CJ, et al. Identification and localization of Huntingtin in brain and human lymphoblastoid cell lines with anti-fusion protein antibodies. Proc Natl Acad Sci U S A. 1995;92:8710-8714.

22. Zuccato C, Valenza M, Cattaneo E. Molecular mechanisms and potential therapeutical targets in Huntington's disease. Physiol Rev. 2010;90:905-981.

23. Ruiz de Almodovar C, Lambrechts D, Mazzone M, Carmeliet P. Role and therapeutic potential of VEGF in the nervous system. Physiol Rev. 2009;89:607-648.

24. McCoy MK, Tansey MG. TNF signaling inhibition in the CNS: implications for normal brain function and neurodegenerative disease. J Neuroinflammation. 2008;5:45.

25. Yamamoto A, Lucas JJ, Hen R. Reversal of neuropathology and motor dysfunction in a conditional model of Huntington's disease. Cell. 2000; 101:57-66.

26. Estrada Sánchez AM, Mejía-Toiber J, Massieu L. Excitotoxic neuronal death and the pathogenesis of Huntington's disease. Arch Med Res. 2007;39(3):265-276.

27. Raymond LA. Excitotoxicity in Huntington disease. Clin Neurosci Res. 2003;3:121-128.

28. Fan MM, Raymond LA. N-methyl-D-aspartate (NMDA) receptor function and excitotoxicity in Huntington's disease. Prog Neurobiol. 2007;81:272-293.

29. Miller BR, Walker AG, Shah AS, Barton SJ, Rebec GV. Dysregulated information processing by medium spiny neurons in striatum of freely behaving mouse models of Huntington's disease. J Neurophysiol. 2008;100:2205-2216.

30. Cepeda C, Wu N, Andre VM, Cummings DM, Levine MS. The corticostriatal pathway in Huntington's disease. Prog Neurobiol. 2007;81:253-271.

31. Huettner JE, Bean BP. Block of N-methyl-D-aspartate-activated current by the anticonvulsant MK-801: selective binding to open channels. Proc Natl Acad Sci U S A. 1987;85:1307-1311.

32. Ayala GX, Tapia R. Late N-methyl-D-aspartate receptor blockade rescues hippocampal neurons from excitotoxic stress and death after 4-aminopyridine-induced epilepsy. Eur J Neurosci. 2005; 22(12):3067-3076.

33. Bates G, Harper P, Jones L. Huntington 's Disease. Oxford, UK: Oxford Univ Press; 2002.

34. Reiner A, Albin RL, Anderson KD, D'Amato CJ, Penney JB, Young AB. Differential loss of striatal projection neurons in Huntington disease. Proc Natl Acad Sci U S A. 1988;85:5733-5737. 
35. Vonsattel JP, Myers RH, Stevens TJ, Ferrante RJ, Bird ED, Richardson EP Jr. Neuropathological classification of Huntington's disease. J Neuropathol Exp Neurol. 1985;44:559-577.

36. Rosas HD, Koroshetz WJ, Chen YI, et al. Evidence for more widespread cerebral pathology in early HD: an MRI-based morphometric analysis. Neurology. 2003;60:1615-1620.

37. Altar CA, Cai N, Bliven T, et al. Anterograde transport of brain-derived neurotrophic factor and its role in the brain. Nature. 1997;389:856-860.

38. Cha JH. Transcriptional signatures in Huntington's disease. Prog Neurobiol. 2007;83:228-248.

39. Chiu E, Alexander L. Causes of death in Huntington's disease. Med J Aust. 1982;1:153.

40. Lanska DJ, Lavine L, Lanska MJ, Schoenberg BS. Huntington's disease mortality in the United States. Neurology. 1988;38:769-772.

41. Gowdak LH, Poliakova L, Wang X, et al. Adenovirus-mediated VEGF(121) gene transfer stimulates angiogenesis in normoperfused skeletal muscle and preserves tissue perfusion after induction of ischemia. Circulation. 2000;102:565-571.
42. Rissanen TT, Korpisalo P, Markkanen JE, et al. Blood flow remodels growing vasculature during vascular endothelial growth factor gene therapy and determines between capillary arterialization and sprouting angiogenesis. Circulation. 2005;112:3937-3946.

43. Desai BS, Schneider JA, Li JL, Carvey PM, Hendey B. Evidence of angiogenic vessels in Alzheimer's disease. J Neural Transm. 2009;116(5):587-597.

44. Desai Bradaric B, Patel A, Schneider JA, Carvey PM, Hendey B. Evidence for angiogenesis in Parkinson's disease, incidental Lewy body disease, and progressive supranuclear palsy. J Neural Transm. 2012;119(1):59-71.

45. Silvestroni A, Faull RL, Strand AD, Möller T. Distinct neuroinflammatory profile in post-mortem human Huntington's disease. Neuroreport. 2009;20:1098-1103.

\section{Publish your work in this journal}

Drug Design, Development and Therapy is an international, peerreviewed open-access journal that spans the spectrum of drug design and development through to clinical applications. Clinical outcomes, patient safety, and programs for the development and effective, safe, and sustained use of medicines are a feature of the journal, which has also been accepted for indexing on PubMed Central. The manuscript management system is completely online and includes a very quick and fair peer-review system, which is all easy to use. Visit http://www.dovepress.com/testimonials.php to read real quotes from published authors.

Submit your manuscript here: http://www.dovepress.com/drug-design-development-and-therapy-journal 